LIPIDS

\section{Picturing palmitoylation}

Science 359, eaao6326 (2018)

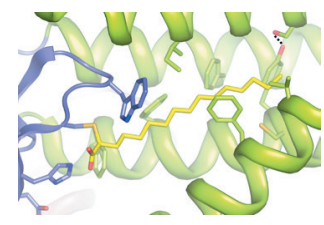

The reversible post-translational attachment of fatty acyl groups to cysteine residues is involved in regulating a range of cellular processes including Ras signaling and intracellular trafficking. The most common acyl chain employed in this modification is the 16-carbon palmitate; its attachment (as palmitoyl-CoA) is catalyzed by polytopic integral membrane enzymes called DHHC palmitoyltransferases, owing to their conserved catalytic motifs. To gain insight into this enzyme's catalytic mechanism and its interactions with its substrates and with the membrane, Rana et al. have now determined the crystal structures of two DHHC enzymes from human and zebrafish. Because the enzyme catalyzes palmitoylation on a substrate protein's membrane-proximal cysteine residues, the active site is situated at the interface between the membrane and aqueous phase. Using the covalent inhibitor 2-bromopalmitate afforded a mimic of the acyl intermediate that is attached to the catalytic cysteine, and the structure of this complex indicates that the acyl chain is stabilized by insertion into an enzyme cavity within the mutagenesis to increase or decrease the cavity size successfully shifted the enzyme's substrate membrane bilayer. Guided by this structure,

preference from palmitoyl-CoA to longer (18-carbon) or shorter (12- and 14-carbon) acyl chains, respectively. In the future, the structures of these DHHC enzymes may be useful for guiding further engineering of orthogonal enzyme-acyl-CoA pairs and for designing new small-molecule probes.

VIRUSES

\section{Capsids under pressure}

Nature doi:10.1038/nature25438 (2018)

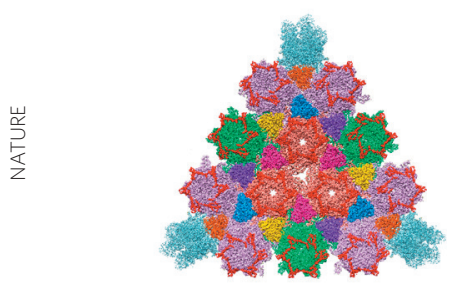

Icosahedral capsids of herpesviruses such as Kaposi's sarcoma-associated herpesvirus (KSHV) consist of nearly 3,000 individual proteins, the bulk of which are pentamers and hexamers of the viral protein MCP decorated with the smaller protein SCP and joined by heterotrimeric triplexes consisting of Tri1 and two Tri2 proteins. To begin to understand how the capsid structure is mechanically stabilized under the high pressure imposed by the double-stranded DNA genome and to gain new insights into capsid assembly, Dai et al. solved the cryo-EM structure of KSHV virions. Extensive analysis of the interactions among the four proteins, including validation with mutagenesis, revealed several critical interactions, including those in which the hydrophobic residues of the L-shaped MCP

UBIQUITINATION

\section{Trashing the CIA}

Mol. Cell 69, 113-125 (2018)

The cytosolic iron-sulfur assembly (CIA) pathway adds an iron-sulfur (Fe-S) cluster to client proteins. One member of the CIA, MMS19, is involved in the transfer of a Fe-S cluster to DNA damage repair enzymes such as XPD. Although the CIA has been characterized for some time, the mechanisms for regulating $\mathrm{CIA}$ activity were not known. Using proteomic approaches, Weon et al. found that MMS19 interacted with a complex composed of the E3 RING ubiquitin ligase NSE1 and its binding partner, MAGE-F1. NSE1 and MAGE-F1 promoted the ubiquitination and degradation of MMS19 by acting on a C-terminal cluster of five lysine residues. MAGE-F1- and NSE1-mediated degradation of MMS19 resulted in impaired ion homeostasis and reduced incorporation of the Fe-S cluster into MMS19 client proteins, leading to a decrease in their overall stability and their ability to efficiently repair DNA damage. Consistent with this, cells expressing high levels of MAGE-F1 exhibited decreased homologous recombination and were highly sensitive to DNA-damaging agents. Finally, genomic analysis revealed that several cancer types exhibited high amplification of MAGE-F1 and were associated with increased genome instability. The findings from Weon et al. reveal a previously unknown connection between ubiquitination and Fe-S clusters with cancer, and future studies could illuminate the exact cellular conditions or upstream components that stimulate MAGE-F1 and NSE1 activity. form a groove with SCP, which itself forms a crosslink to a neighboring MCP and also supports $\pi$-stacking interactions between MCP and SCP. The authors found the MCP hydrophobic groove to be targetable with a peptide mimic that reduces virion production. Furthermore, $\beta$-strands between adjacent MCPs join together to form, via disulfide bonding, a turbine-shaped ring in each hexon. The authors also defined three types of network interactions in MCP that are critical for the mechanical sturdiness of the KSHV capsid, as well as interactions with the Tri proteins critical for capsid assembly. These results highlight a complex assembly mechanism optimized for stability against the pressure imposed by the DNA contained within.

GLYCOSYLATION

\section{Gluing on glycans}

Science doi:10.1126/science.aar5140 (2018)

Oligosaccharyltransferase (OST) catalyzes the key step during N-linked glycosylationthe transfer of the precursor glycan from its lipid carrier to the Asn side chains of substrate proteins. Whereas eukaryotes contain a multisubunit OST localized to the endoplasmic reticulum, which recognizes the Asn-X-Ser/Thr motif in secretory proteins, bacteria encode a plasma-membrane-bound single-subunit OST (ssOST) that is specific for substrates with an Asp-X-Asn-X-Ser/ Thr sequon. Wild et al. isolated intact OST from yeast and determined the 3.3- $\AA$ cryoEM structure of the nanodisc-reconstituted complex. The structure revealed that the active site of the OST catalytic subunit STT3 is surface exposed, allowing unhindered substrate access. In addition, differences in the shape and charge distribution between the catalytic sites of STT3 and bacterial ssOST explain the broader substrate specificity of eukaryotic OST. The other seven subunits of the yeast OST complex have scaffolding functions, and their luminal domains may serve as docking platforms for interaction partners and promote substrate binding. The authors also fitted their structure into an earlier published lower-resolution cryo-tomography map of the mammalian ribosome-translocon-OST supercomplex, showing how the nascent polypeptide chain emerging from the luminal exit of the translocon can access the OST active site. The first high-resolution eukaryotic OST structure provides mechanistic insights into $\mathrm{N}$-linked glycosylation and will promote further structural and functional studies. 